\title{
Nanoscale
}

Check for updates

Cite this: Nanoscale, 2019, 11, 11402

\section{LEDs using halide perovskite nanocrystal emitters}

\begin{abstract}
Fei Yan (D) a and Hilmi Volkan Demir (D) *a,b,c
The emerging family of lead-halide perovskite (LHP) nanocrystal emitters has shown impressive achievements in solid-state light-emitting applications. With luminous efficiency comparable to that of organic light-emitting diodes, LHP light-emitting diodes (PeLEDs) have demonstrated a wide colour gamut with high colour purity and a widely tunable range of emissive wavelengths across the whole visible range. Herein, the understanding of LHP nanocrystals in light emission and the resulting PeLEDs are reviewed. Additionally, key features of LHP nanocrystal emitters applied in PeLEDs and guidelines towards realizing high-performance devices are discussed.
\end{abstract}

\section{Introduction}

From rock painting to augmented reality, numerous display technologies have been developed to illustrate the colourful world. Intuitive drawing is the predominant method of vision for apperceiving information from the world around us. In such a messaging process, the truthfulness of information, including colour data, is essential. Therefore, various fullcolour display technologies from cathode-ray tubes to liquidcrystal displays (LCDs) and then to flexible organic light-emitting diodes (OLEDs) have emerged. However, no available display technologies can fully cover the natural colour gamut as recognized by the National Television System Committee (NTSC) TV colour standard (Fig. 1a). Recently, highly efficient lead-halide perovskites (LHPs) have emerged as a new kind of solid-state emitters, and the resulting perovskite light-emitting diodes (PeLEDs) have achieved performance levels comparable to OLEDs and cadmium selenide (CdSe) colloidal quantum dot light-emitting diodes (QLEDs), including high luminous efficiency, low cost, large area, and flexibility. ${ }^{1-24}$

Most efficient emitters used in PeLEDs are LHP nanocrystals (NCs), for which films reach a near-unity photoluminescence quantum yield (PLQY) by facilitating exciton radiative recombination $;^{20,25-30}$ the resulting PeLEDs have achieved an external quantum efficiency (EQE) of over $20 \% .^{21-24}$ Moreover, because of the high colour purity and

${ }^{a}$ LUMINOUS! Centre of Excellence for Semiconductor Lighting and Displays, TPI-The Photonics Institute, School of Electrical and Electronic Engineering, Nanyang Technological University, 639798, Singapore.

E-mail: HVDEMIR@ntu.edu.sg

${ }^{b}$ Division of Physics and Applied Physics, School of Physical and Mathematical Sciences Nanyang Technological University, 639798, Singapore

${ }^{c}$ Department of Electrical and Electronics Engineering, Department of Physics, UNAM-Institute of Materials Science and Nanotechnology, Bilkent University, Ankara 06800, Turkey broad emissive wavelength range, PeLEDs demonstrate an unprecedented colour gamut, which almost completely cover the CIE 1931 colour spaces (Fig. 1a and b). ${ }^{31-34}$

The LHP NCs can be indicated in a chemical formula $(\mathrm{R})_{2}(\mathrm{~A})_{n-1} \mathrm{~B}_{n} \mathrm{X}_{3 n+1}$, where $n=\infty$ corresponds to the normal $3 \mathrm{D}$ LHPs with chemical formula $\mathrm{ABX}_{3}$ and $n=1$ corresponds to the monolayer 2D LHP $\mathrm{R}_{2} \mathrm{ABX}_{4}$ (Fig. 1c). ${ }^{35}$ Normally site $\mathrm{A}$ is an organic cation of formamidinium (FA), methylammonium (MA) or $\mathrm{Cs}^{+}$; site $\mathrm{B}$ is $\mathrm{Pb}^{2+}$; site $\mathrm{X}$ is $\mathrm{Cl}^{-}, \mathrm{Br}^{-}$, or $\mathrm{I}^{-}$; and $\mathrm{R}$ is a molecule spacer in the $2 \mathrm{D}$ LHPs. ${ }^{35-39}$ The corner-sharing $\left[\mathrm{PbX}_{6}\right]^{4-}$ octahedra are the core of LHPs, which surround the cations to compose LHPs with a 3D structure or sandwiched by a molecular spacer to form LHPs with a 2D structure. ${ }^{35-39}$ By changing the halide component from iodine to chlorine, the emissive spectra can be adjusted significantly from the near infrared to near ultraviolet (Fig. 1b). ${ }^{35-39}$

In this review, we summarized up-to-date achievements for PeLEDs using LHP NCs, also including quantum dots (QDs) and nanoplatelets (NPLs) with strong quantum confinement, we also present insights on device work, including physics of light emission and device fabrication, and guidelines for high performance PeLEDs for the readers. We envision that this review will trigger some subsequent works or new possibilities for PeLEDs and other relative subfields.

\section{Fundamental properties of LHP nanocrystals as efficient emitters}

With a low binding energy of dozens of meV caused by a large dielectric constant, the Wannier-Mott excitons formed in LHPs dissociate into free charge carriers with a high possibility at room temperature, which is one merit for superior performance photovoltaics. ${ }^{40-43}$ With the high electron and hole mobility in LHPs, the charge carriers are almost free with neg- 

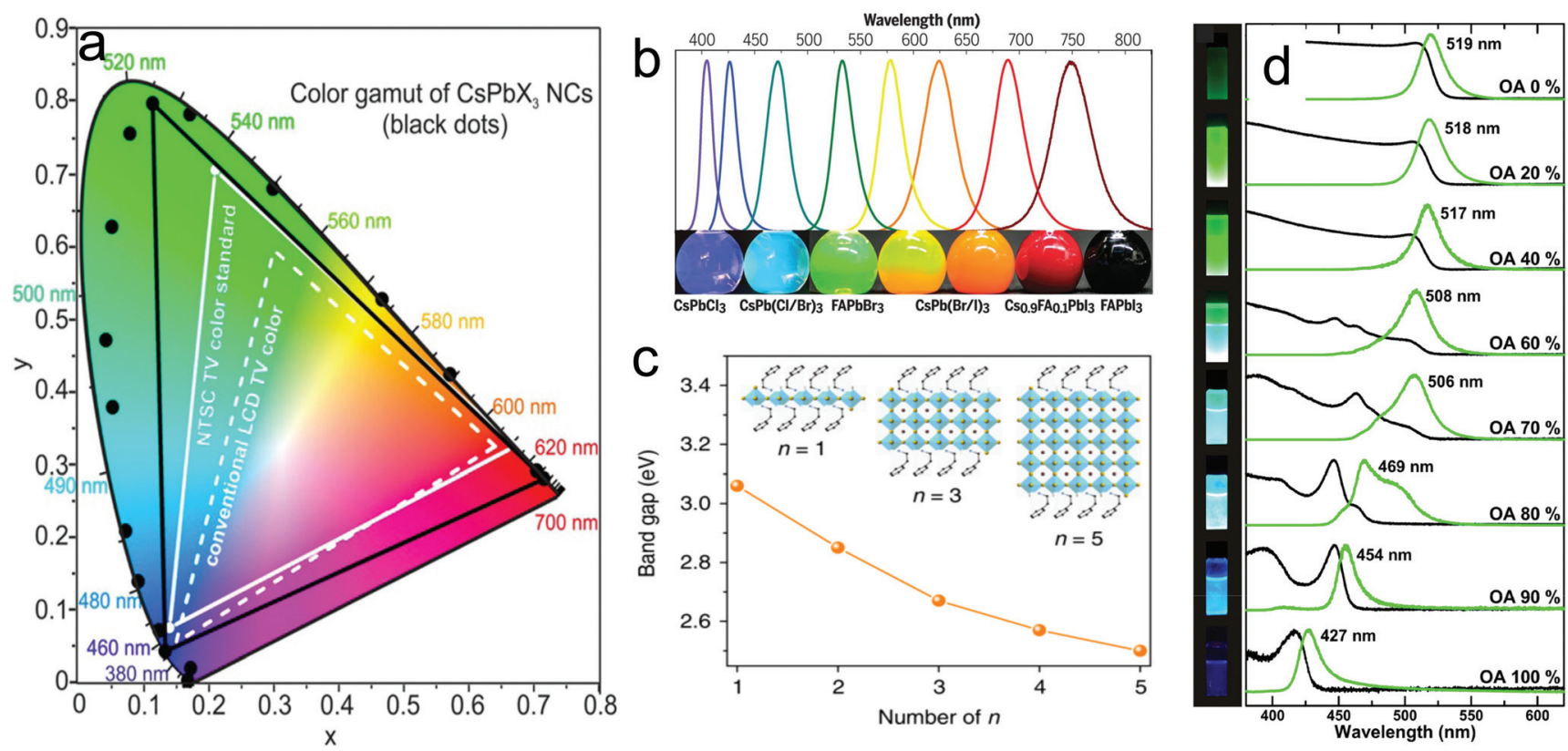

Fig. 1 (a) CIE chromaticity coordinates (dark points) for $\mathrm{CsPbX}_{3}$ LHP NCs emission compared with commercialized LCD TVs (dashed white line) and

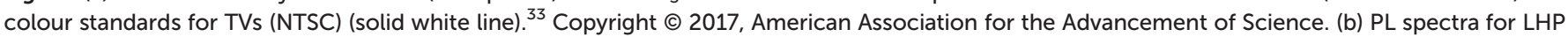
NCs with different halide components. ${ }^{33}$ Copyright $\odot 2017$, American Association for the Advancement of Science. (c) Schematic and bandgap of quasi-2D perovskite with a varying number of layers. ${ }^{35}$ Copyright $\odot$ 2018, Springer Nature. (d) Photographs of the LHP NPL suspensions under UV light. ${ }^{39}$ Copyright $\odot 2015$ American Chemical Society.

ligible wavefunction overlap. ${ }^{44,45}$ Generally, the radiation from LHP polymorph emitters mainly stems from a slow bimolecular recombination with an intrinsic constant determined by the hole-electron cross-section and group velocities (Fig. 2a). ${ }^{46,47}$ Similarly, it is supposed that the exciton formed by injected carriers (in electrical excitation) also dissociate back to free charge carriers with a high possibility, which limits the luminescence efficiency of PeLEDs. In LHP NCs with dimensions in the range of several nanometres to tens of nanometres, the charge carriers are confined in a small-sized domain by the grain boundary and passivate ligands, which increases the exciton biding energy by up to approximately $300 \mathrm{meV}^{48-50}$ Owing to the comparable effective mass of the hole and electron, the confinement of their wavefunctions are almost equal, which facilitates efficient first-order exciton recombination dominating the two-order bimolecular recombination (Fig. 2a). ${ }^{20,51-56}$

As a highly ionic compound, normally, LHP NCs are highly crystalline with a low level of defects. ${ }^{57}$ The electronic band gap for LHP NCs is determined by the difference between the antibonding orbitals of $\mathrm{Pb}_{6 \mathrm{~s}}-\mathrm{X}_{n \mathrm{p}}$ couplings (bottom of conduction band) and antibonding orbitals of $\mathrm{Pb}_{6 \mathrm{p}}-\mathrm{X}_{n \mathrm{p}}$ couplings (top of valence band) $\left(\mathrm{X}_{n \mathrm{p}}: \mathrm{Cl}_{3 \mathrm{p}}, \mathrm{Br}_{4 \mathrm{p}}, \mathrm{I}_{5 \mathrm{p}}\right) \cdot{ }^{57-59}$ In addition, their bonding orbitals are deeper within the valence band (Fig. 2b). ${ }^{57-59}$ Therefore, such a special electronic band structure results in an intrinsic defect tolerance by leaving a clean band gap free of deep trap states (Fig. 2b), which is unlike the band gap formed by the antibonding conduction band and bonding valence band in conventional semiconductors. ${ }^{57-59}$
Thus, in virtue of a low level of trap-mediated non-radiative recombination, the LHP NCs exhibit high PLQY emission in colloidal solution even without any passivation of ligands. ${ }^{33,58,61,62}$ Furthermore, the surface defects in LHP NCs can be effectively passivated by capping ligands, which enhances the PLQY of LHP NCs by up to near unity. ${ }^{60,63-66}$

Although the LHP NCs exhibit an efficient exciton emission with a high exciton binding energy of up to approximately $300 \mathrm{meV}$, which is close to the value for an organic molecule exciton, the excitons, however, are not unambiguously of the Frenkel or Wannier type. ${ }^{48-50,67}$ it is believed that the exciton is a hybrid of the Frenkel and Wannier types in 2D structure LHPs. ${ }^{67}$ Unlike organic molecule exciton emission that follows a spin selection rule, by virtue of strong spin-orbital coupling, which is supposed to be related to the heavy elements contained therein, the spin angular momentum conservation is not a transition selection rule that limits the luminous efficiency of LHP NCs. ${ }^{68}$ Moreover, unlike other inorganic semiconductors, e.g., CdSe QDs, for which the lowest state of the exciton is dipole-forbidden, the emission from $\mathrm{CsPbX}_{3}$ NCs was demonstrated to originate from the lowest state of the exciton. ${ }^{68}$ With three bright triplet states from four sublevels, the $\mathrm{CsPbX}_{3}$ NCs indicate a fast exciton radiation (Fig. 2c). ${ }^{68}$ Therefore, the LHP NCs demonstrate near unity quantum yield in both optical and electrical excitations. ${ }^{21-24}$

Due to the predominant role of $\mathrm{Pb}$ and $\mathrm{X}$ in determining the conduction band and valence band, respectively, the band gap can be tuned by changing the halogen species from $\mathrm{Cl}$ to I, leading to an adjustable emission wavelength through the 

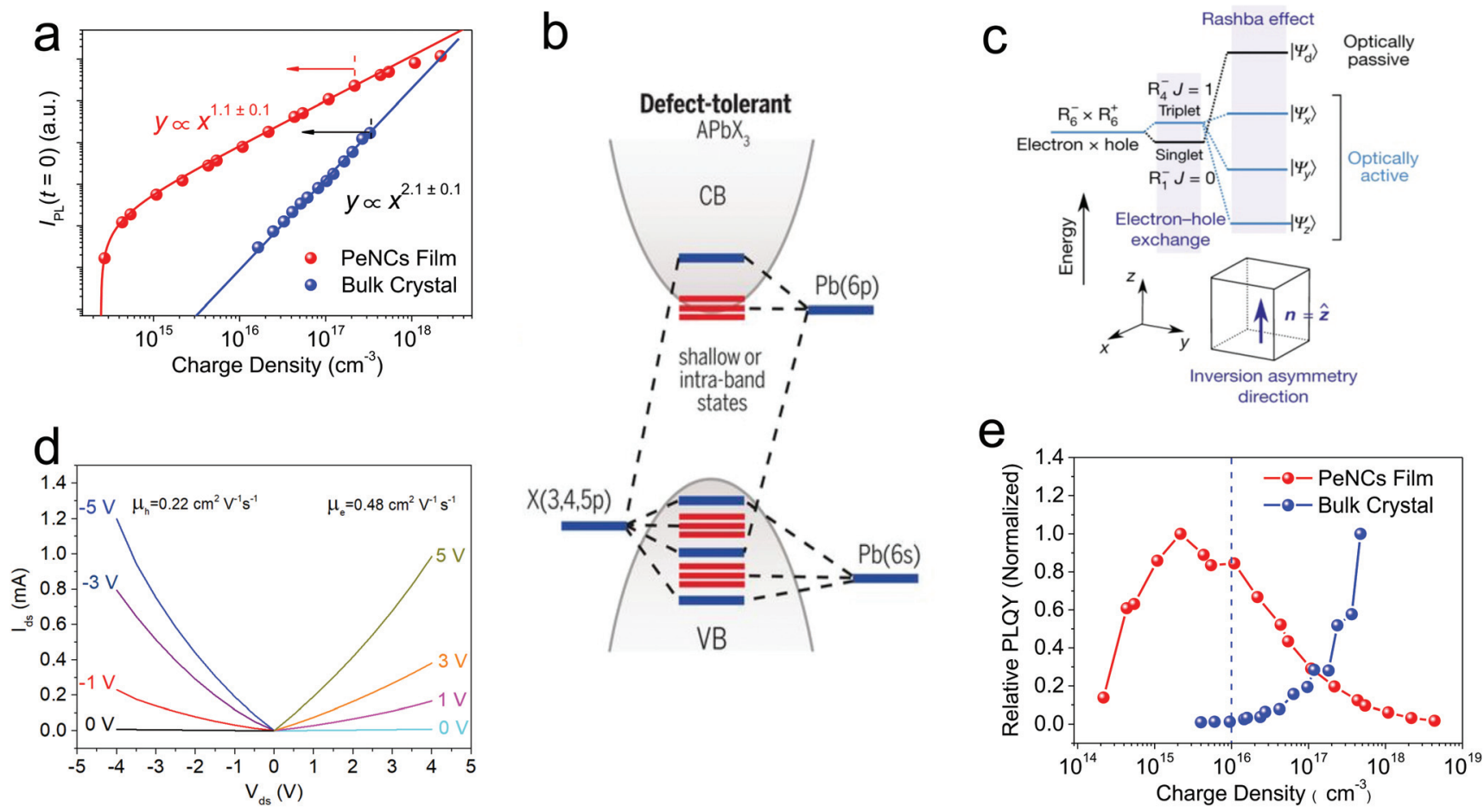

Fig. 2 (a) Photon-injected charge carrier density dependence of the initial-time PL intensity (IPL $(t=0)) .{ }^{20}$ Copyright $\odot 2018$ American Chemical Society. (b) Schematic of the band structure and defect-tolerant electronic structure of LHPs. ${ }^{33}$ Copyright @ 2017 American Association for the Advancement of Science. (c) The expected fine structure for the $\mathrm{CsPbBr}_{3}$ exciton. The exciton splits into three bright states and a higher-energy dark state. ${ }^{68}$ Copyright $\odot$ 2018, Springer Nature. (d) Mobility levels for the electron and hole derived from a field-effect transistor using $\mathrm{MAPbBr}_{3}$ PeNCs film as the active layer. ${ }^{20}$ Copyright (c) 2018 American Chemical Society. (e) PLQY as a function of the photon-injected charge carrier density. ${ }^{20}$ Copyright $\odot 2018$ American Chemical Society.

whole visible range. ${ }^{57-59}$ Furthermore, using mixed halogen species to take site $\mathrm{X}$, the emissive wavelength of LHPs can be flexibly controlled in the visible range (Fig. 1b). ${ }^{35,69-73}$ Compared to OLEDs and QLEDs, the LHP NCs and their PeLEDs demonstrate a much narrower linewidth for the emission band. ${ }^{1-24}$ In hybrid LHPs with polar bonds, the narrow emissive band is believed to be mainly determined by the Fröhlich interaction between longitudinal optical phonons and charge carriers. ${ }^{74-76}$ Moreover, owing to the great defect tolerance of LHP NCs, the broadening of the emission spectrum induced by traps is at a negligible level. ${ }^{58,59,77}$ These features endow the LHP NCs with a high colour purity, which is another essential characteristics for a full colour display. Combining the flexible tunability of the emission wavelength through the whole visible range and high colour purity, the LHP NCs demonstrate a broad colour gamut, which almost overlaps the whole CIE 1931 colour space (Fig. 1a). ${ }^{31-33}$ Though the phase separation in LHP NCs with a mixed halide caused by ion migration results in a low stability for the emission spectrum under electrical excitation, ${ }^{78-80}$ the RGB primary colour LHP NCs with a single halide exhibit stable EL spectra, which can be used to compose almost all target colours in the visible range (Fig. 1a). ${ }^{31-33}$

Bromide-based green and iodide-based red PeLEDs have achieved comparable EQE values as OLEDs and QLEDs. ${ }^{21-24}$
However, chloride-based blue LHP NCs still present a low luminous efficiency level in PeLEDs, for which the underlying reasons are the low intrinsic luminous efficiency and emission shift caused by phase separation under electrical excitation. ${ }^{35,78,81}$ With a small exciton Bohr radius, which is close to the dimension of the $\left[\mathrm{PbBr}_{6}\right]^{4-}$ octahedral cell, it is difficult to obtain a qualified blue emission from $3 \mathrm{D} \mathrm{APbBr}_{3}$ NCs with strong quantum confinement by controlling the particle size. Alternatively, $\mathrm{APbBr}_{3}$ multilayer NPLs with strong quantum confinement can emit qualified blue light, as required for the blue component in a full colour display. ${ }^{35,82-84}$ By controlling the amount of $\left[\mathrm{PbBr}_{6}\right]^{4-}$ in the layers, the emission colour from LHP NPLs can be adjusted from a deep blue to sky blue (Fig. 1d), ${ }^{35,82-84}$ and due to the quantized amount of $\left[\mathrm{PbX}_{6}\right]^{4-}$ cell, it is easy to obtain a high mono-dispersion of LHP NCs, resulting in a narrow emissive spectrum.

With the large polaron having minimized charge carrier scattering with defects, other charge carriers, and longitudinal optical phonons, LHP NCs exhibit high carrier mobility even in nanocrystals with confinement generated by the grain boundary and passivated ligands (Fig. 2d). ${ }^{20,85}$ With small and comparable effective masses, ${ }^{56}$ generally, the high hole mobility is comparable to that for electrons, which is also an important merit for achieving balanced charge carriers in the recombination zone of efficient PeLEDs (Fig. 2d). ${ }^{20,85}$ However, such 
high charge carrier transport involves electron-ion mixed conduction, and directional ion-migration driven by the external electrical field leads to the degradation of LHP NCs. ${ }^{86,87}$

\section{Classification of LHP nanocrystal emitters}

Although defects can be tolerated in LHPs, the non-radiative losses are still present at a substantial level, which suggests that un-neglected deep states exist in the bandgap (Fig. 2e). Such traps quench the luminescence through a first-order Shockley-Read-Hall non-radiative recombination at a comparable rate to the exciton radiative process. $^{20,40,42}$ Therefore, most LHP nanostructured emitters are crystalline surrounded by passivating ligands to minimize trap states. ${ }^{10-24}$ Although having similar components and formulae, LHP NCs with different structures, e.g., 3D and 2D, and morphology exhibit some individual features in terms of light emission. ${ }^{31-33}$ In addition, LHP NC films prepared in colloidal solution also demonstrate some differences compared to a film deposited in situ using a precursor solution. ${ }^{20-24,35}$

Normally, LHPs NCs have a 3D structure, which is similar to the bulky counterpart. By decreasing the dimension of the LHP NCs to a size comparable to the exciton Bohr radius, the quantum confinement effect emerges, and with strong quantum confinement the emission from LHP NCs exhibits a significant blueshift, ${ }^{21,88}$ even to the blue range. ${ }^{35,82}$ However, as the size of the exciton Bohr radius is comparable to the $\left[\mathrm{PbX}_{6}\right]^{4-}$ cell, it is difficult to precisely control the particle size in 3D structure LHP QDs, which may lead to a broadening of the emissive spectrum. ${ }^{21,88}$ Due to the small size and light weight of LHP QDs, post-treatments, e.g., purification and concentration, of the primary colloidal solution becomes difficult.

Alternatively, by decreasing the layer number for the $\left[\mathrm{PbX}_{6}\right]^{4-}$ cell, the emission from LHP NPLs also exhibits a blueshift with enhancement of quantum confinement. With a thickness of approximately $1 \mathrm{~nm}$, monolayer LHP NPLs demonstrate an emission with a significant blueshift of approximately $400 \mathrm{meV}$ compared to the 3D counterparts (Fig. 1c and d). ${ }^{82}$ In addition, as the amount of $\left[\mathrm{PbX}_{6}\right]^{4-}$ layer sandwiched by molecule spacers in LHP NPLs is quantized, the emission spectra can be adjusted more precisely compared to the LHP QDs. ${ }^{35,39,82-84,89-91}$ Similar to the 2D counterparts, the LHP NPLs also show a better stability by protecting the $\left[\mathrm{PbX}_{6}\right]^{4-}$ core against ambient moisture and oxygen; however, the interlayer charge carrier transport also becomes more difficult in LHP NPLs compared to 3D LHP NCs. ${ }^{35,39,82-84,89-91}$ Moreover, due to the $2 \mathrm{D}$ exciton feature and the diminished dielectric screening, the radiation from excitons in LHP NPLS is faster compared to the 3D LHP NCs. ${ }^{68}$

Alternatively, in situ fabrication using precursor solution is another approach for depositing LHP NCs films with the assistance of passivating ligands. Similarly, some matrix polymers can be mixed into the precursor solution for controlling the NC dimensions during the film deposition (Fig. 3a and b). ${ }^{92}$
With the consideration of environmental friendliness, lead-free perovskite emitters using $\mathrm{Sn}, \mathrm{Ge}, \mathrm{Sb}, \mathrm{In}, \mathrm{Ag}$, and $\mathrm{Bi}$ instead of $\mathrm{Pb}$ with an electron structure or crystallographic structure that replicates halide perovskites have been studied. ${ }^{93-97} A_{3} B_{2} X_{9}$ halide perovskites give efficient emission from the blue to UVA, which can be regarded as potential blue emitters for PeLEDs. ${ }^{98,99}$ With a broad-band spectrum, a leadfree double perovskite $\mathrm{Cs}_{2} \mathrm{Ag}_{0.60} \mathrm{Na}_{0.40} \mathrm{InCl}_{6}$ exhibits a warm white emission with a PLQY of approximately 90\% (Fig. 3c), which suggests that white-colour PeLEDs with a simple structure are possible. ${ }^{97}$ However, so far, lead-based perovskite emitters have dominated the highest performance in PeLEDs. ${ }^{21-24,93-99}$

\section{Key features of LHP nanocrystals in LEDs}

As an ionic compound, most LHP emissive layers, including the nanocrystal emitter film, in PeLEDs have been deposited using solution processing, which constrains the design of the device structure and the adoption of other functional layer materials. The deposited film must be tough enough against subsequent film fabrication; also a lower surface tension solution is required for good wetting onto the substrate. Due to the highly ionic bonding of the LHPs, generally, a solvent with high dielectric constant, such as alcohol, is excluded from device fabrication, as it can damage LHPs. ${ }^{57,100}$

Due to the low lying energy levels of LHP NCs, there is a high energy barrier for hole injection into the emissive layer (Fig. 3d). ${ }^{20-23}$ Due to the dominating role of $\mathrm{X}$ in determining the valence band, by changing the halide from iodide to chloride, the extension of the band gap mainly occurs to the valence band, leading to a higher barrier for hole injection (Fig. 2b). ${ }^{57-59}$ Sometimes, a buffering layer can be used to increase hole injection. ${ }^{16,21}$ As the directional migration of ion species inside a LHP layer is driven by the applied electrical field, p-type and n-type doping layers are formed at corresponding interfaces, which facilitates charge carrier injection into the LHP layer (Fig. 3e). ${ }^{87,101}$ That is why some PeLEDs can be driven by a low voltage even without any assistance of hole injection layers (Fig. 4a). ${ }^{20,22,101}$ However, such low driving voltage is mostly accompanied by the shrinking of the LHP layers due to the decomposition of emitters, leading to a drop in the luminous efficiency and short device operation lifetime. ${ }^{87,101}$

The PLQY of LHP NC films plays a major role in determining the maximum EQE that can be reached by the resulting PeLEDs. To suppress the trap-mediated non-radiative recombination, high-ratio ligands are generally required for passivating the surface defects in LHP NCs, which also ensures good stability of the colloidal solution by preventing aggregation of the LHP NCs and smoothening the morphology of the resulting emissive film. ${ }^{1-16,20-23}$ Similarly, for in situ deposition using a precursor solvent, high-ratio ligands are also used for controlling the LHP NC size and film quality. ${ }^{35}$ However, most passi- 

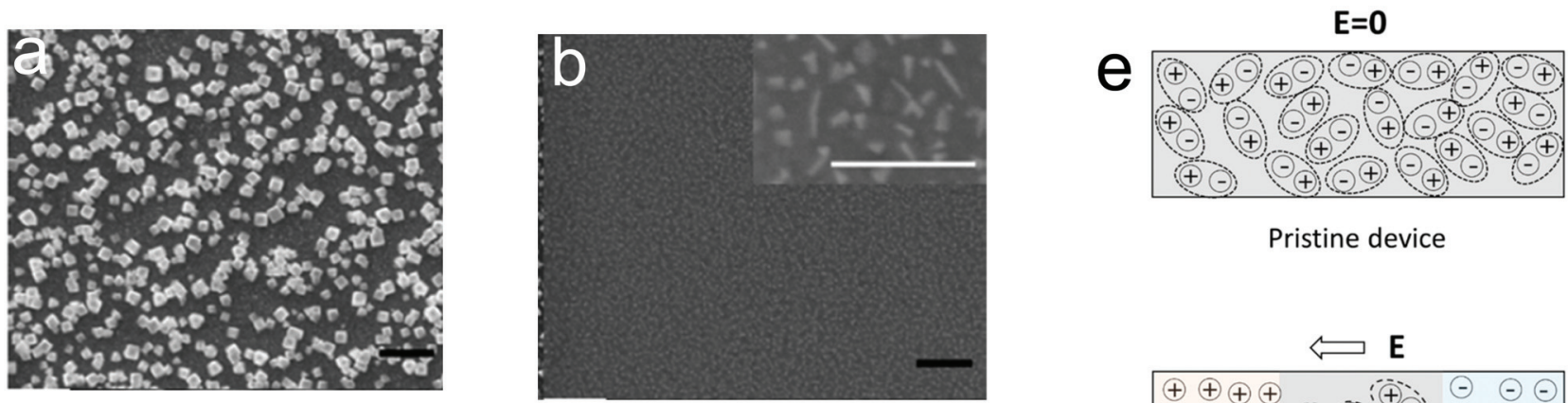

Pristine device
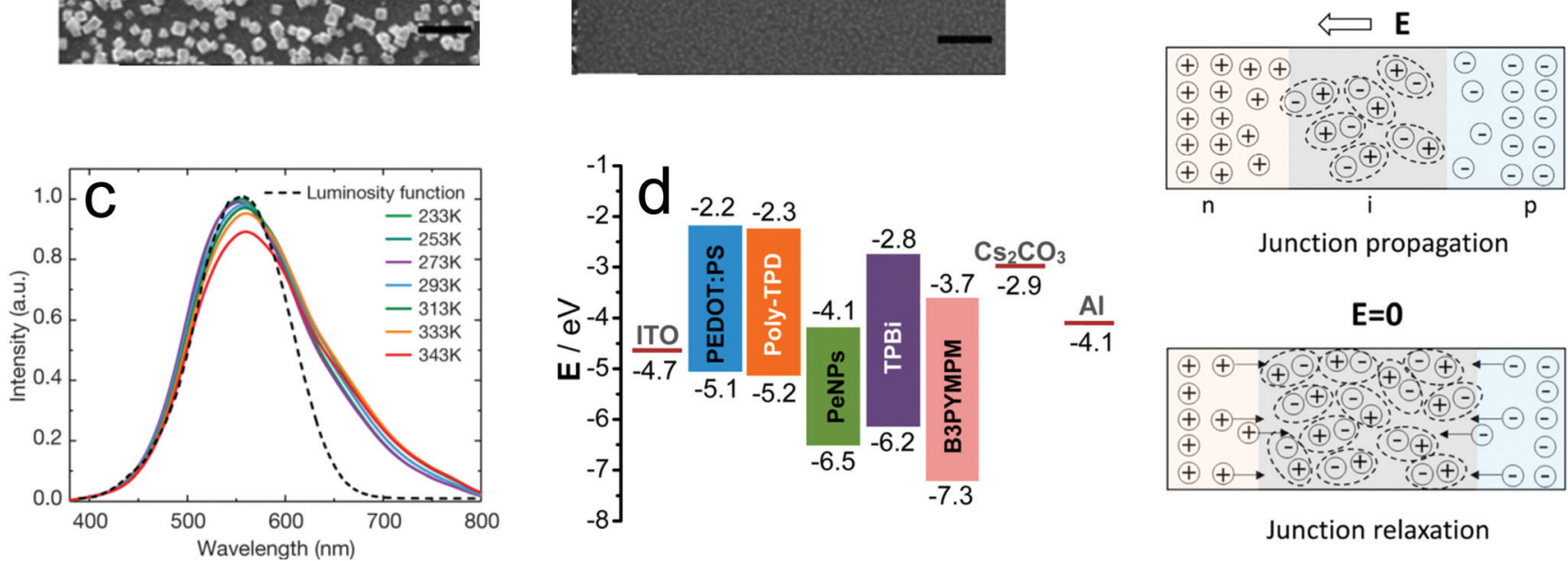

Junction propagation

$E=0$

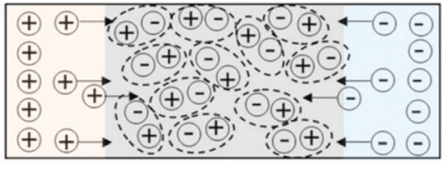

Junction relaxation

Fig. 3 SEM image of (a) $\mathrm{MAPbBr}_{3} \mathrm{NCs}$ only, (b) 1/2 polyimide/MAPbBr 3 on a PEDOT:PSS film. Inset in (b) shows an enlarged image of a 1/2 polyimide/ $\mathrm{MAPbBr}_{3}$ film. ${ }^{92}$ Copyright () 2015 American Chemical Society. (c) Luminosity function and photoluminescence spectra for $\mathrm{Cs}_{2} \mathrm{Ag}_{0.60} \mathrm{Na}_{0.40} \mathrm{InCl}_{6}$ measured at different temperatures. ${ }^{97}$ Copyright $\odot 2018$ Springer Nature. (d) Energy level alignment for a PeLED. ${ }^{16}$ Copyright $\odot$ 2016 American Chemical Society. (e) Distribution of ions in a pristine film, a film under an external electrical field, and a relaxed film after removal of the bias. ${ }^{101}$ Copyright @ 2017 American Chemical Society.
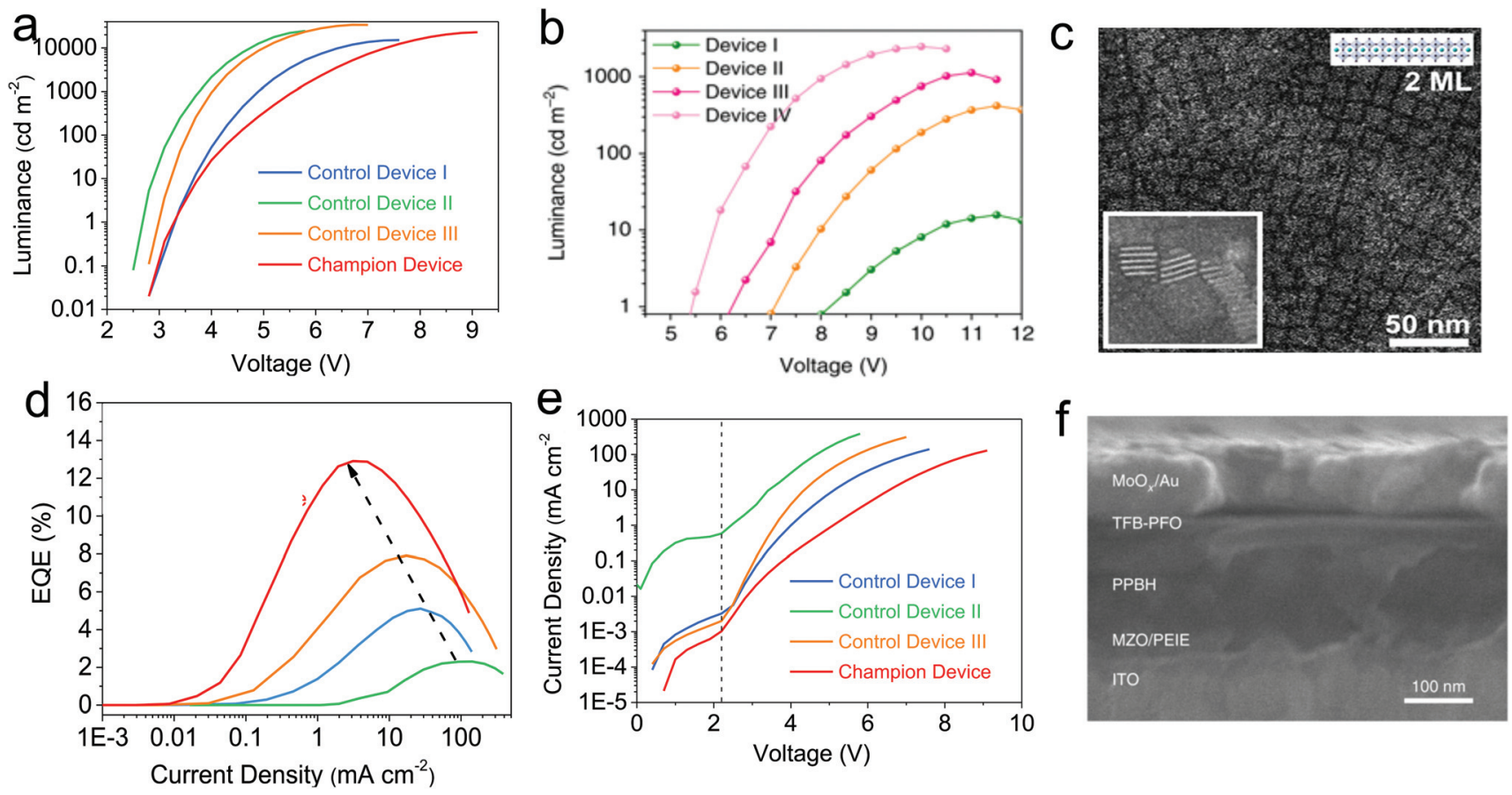

Fig. 4 (a) Luminance-voltage dependence for devices using LHP NC emitters. ${ }^{20}$ Copyright $\odot 2018$ American Chemical Society. (b) Luminancevoltage dependence for devices using LHP NPL emitters. ${ }^{35}$ Copyright $\odot$ 2018, Springer Nature. (c) 2 monolayer LHP NPLs and stacked structure (inset). ${ }^{82}$ Copyright $\odot 2018$ American Chemical Society. (d) EQE-current density characteristics and (e) $I-V$ curves for PeLEDs with MAPbBr 3 NC emitters. ${ }^{20}$ Copyright $\odot 2018$ American Chemical Society. (f) Cross-sectional scanning electron microscopy image of the LED structure using a perovskite-polymer emitter. Copyright $\odot 2018$, Springer Nature. ${ }^{23}$ 
vating ligands, such as oleylamine, are insulating for charge carrier injection into the surrounding LHP NCs during electrical excitation due to the long-chain alkyl. ${ }^{1-30}$

In LHP NPLs, the layer-by-layer structure of LHP NPLs sandwiched by a molecule spacer also blocks the vertical charge carrier transport, which leads to a much higher energy barrier for charge carrier injection into $\left[\mathrm{PbX}_{6}\right]^{4-}$ recombination centres (Fig. 4b). ${ }^{35,39,82}$ Due to the unstable and dynamic binding of capping ligands to LHP NCs, the prepared colloidal solution suffers a degradation caused by the aggregation of LHP NCs, leading to a decreased luminous efficiency and a rough morphology for the deposited film. ${ }^{57,100}$ Specially, because of the larger lateral size compared to the thickness, the LHP NPLs in colloidal solution stack together in the vertical direction (Fig. 4c). ${ }^{57,86,100}$ The lower stability of the colloidal solution constrains the time window for subsequent film deposition in applications, e.g., PeLEDs, which require a high quality film. For in situ precursor solution deposition, the most common solvents adopted are dimethyl sulfoxide (DMSO) and dimethylformamide (DMF), which are difficult to wet on most polymer substrates for LHP NC film deposition due to the relatively higher surface tension. ${ }^{35,102,103}$ Some treatments, such as oxygen plasma, for the prepared polymer substrates make them negatively charged and can be used to enhance the wetting of the precursor solutions. ${ }^{102,103}$

\section{Guidelines for high-performance PeLEDs}

The emission from LHP NCs originates from the radiative recombination of an exciton formed by the injected hole and electron in PeLEDs. As the most important parameter for characterizing PeLEDs, a high EQE means maximized photon generation based on minimized electron injection into PeLEDs. Obviously, the EQE loss is mainly caused by inefficient photon generation and waste of injected charge carriers. First, to reach an efficient photon generation, the loss caused by trap-mediated non-radiative recombination needs to be minimized, thus a high-quality LHP NC film with reduced defects is essential. ${ }^{20-24}$ Second, if the ratio of electrons to holes injected into the recombination zone deviates from unity, the redundant charge carriers themselves are lost, which can also quench the exciton radiative recombination through the non-radiative Auger process. ${ }^{20-24}$ Even with balanced charge carriers in the recombination zone, Auger recombination still plays a significant role in a fall in EQE at high driving current density levels. To reduce the EQE loss caused by leakage current without recombination, which requires most injected charge carriers to flow through the nanostructured emitters, the LHP NC film needs to be uniform and continuous because otherwise the pinholes provide a bypass for charge carriers crossing the emissive layer without recombination. Some additives or a post-treatment can be applied to enhance LHP NC film quality. ${ }^{22,23}$
Normally, the EQE reaches a maximum value at a relatively low driving current density level, and then drops with increasing driving current (Fig. 4d). ${ }^{20-23,35}$ It is then impossible to avoid the combination of an Auger process, Joule heating, imbalanced charge carriers, emitter decomposition, and other factors. It is reasonable to sustain a high EQE at a low driving current density level because of the negative influences caused by Joule heating, emitter decomposition, and Auger non-radiative recombination on EQE remain at a low level (Fig. 4d). ${ }^{20-23}$ Moreover, because of the relatively higher ratio at low driving current density levels, a lower un-recombined leakage current is required. Such un-recombined leakage current levels are indicated by the current density level when the bias is lower than the threshold value, which is also an important parameter for characterizing the device quality (Fig. 4e). Though the mobility of electrons is comparable to that of holes for an $\mathrm{LHP}^{20}$ mobility values in other organic functional layers are normally different and electric field dependent, ${ }^{104,105}$ which means that it is difficult to ensure charge carrier balance under varying driving voltage. Hence, a high quality device is required to maintain balanced charge carriers in the recombination zone at a low driving current density level.

The maximum brightness, an extensive parameter, which a PeLED can achieve depends on the amount of excited emitters, which suggests that a thick LHP NC film is required. However, due to the efficiency loss caused by self-absorption of LHP NCs emitters, normally, an emissive film with an optimized thickness of dozens of nanometres is essential. ${ }^{5,20-24}$ As a currentdriven device, the brightness of PeLEDs is proportional to the driving current in the normal working range, and a larger slope indicates a higher EQE. Thus, the higher the maximum driving current density that a PeLED can achieve before device degeneration, the higher the maximum brightness it can output. Especially, as the amplified spontaneous emission is normally observed at a high excitation density level, a high maximum driving current density is essential for achieving electrically pumped lasing. ${ }^{106}$ Therefore, it is required that the charge carrier mobility and energy level alignments for all the functional layers match well with the LHP NC layer. It is a tough challenge for most organic charge carrier transport materials to support such a high density of driving current because of low charge carrier mobilities. ${ }^{104,105}$ The Joule heating originating from high resistance under a high density of driving current leads to a degradation of the organic molecule films. Normally, inorganic semiconductors indicate higher charge carrier mobility; however, the higher density of charge carriers can quench the emission from LHP NCs, and a high-temperature deposition procedure can also damage LHP $\mathrm{NCs}^{24}$ Due to the energy barrier, the charging caused by carrier accumulation at the interface needs to be eliminated using buffer layers.

Under an applied field, the LHP NCs suffer degradation caused by ion migration. ${ }^{87,101}$ Therefore, insulating (e.g., PMMA) or semiconducting polymer thin films and a matrix, can be adopted for constraining ion migration in LHP NCs, while also providing protection against ambient moisture and 
oxygen (Fig. 4f). ${ }^{22,23,92,107,108}$ Compared to inorganic materials, the processing of an organic film or matrix is easier, which lowers the charge carrier density, leading to a weak interfacial quenching. ${ }^{22,23}$ Additionally, an organic semiconducting layer with matched energy levels can increase the charge carrier injection into an LHP emissive layer.

The metallic PEDOT:PSS electrode with a work function of around $-4.7 \mathrm{eV}$ is also considered to be a quencher for excitons, thus a hole transport layer (HTL), such as poly[ $N$, $N^{\prime}$-bis(4-butylphenyl)- $N, N^{\prime}$-bisphenylbenzidine] (poly-TPD), is adopted before the deposition of an LHP NC film (Fig. 3d). ${ }^{16,21}$ In addition, an organic film with low charge carrier density is used as a buffering layer to prevent potential quenching caused by the PEDOT:PSS film, which is supposed to be a metallic electrode modification layer even electrode. ${ }^{16,21,109}$ Additionally, such an HTL with a high-lying LUMO is supposed to serve as an electron blocking layer to confine the electron migration by suppressing the leakage overflow current. However, some PeLEDs without any buffer layer between the PEDOT:PSS and LHP emissive layers can still demonstrate a comparable performance level, even showing an EQE of over 20\%, compared to devices with an HTL. ${ }^{20-22}$ The PeLEDs without an HTL also present a high device quality with small leakage current at low and high driving current density level (Fig. 4d). ${ }^{20-22}$ It seems that the organic HTL is not essential for realizing high-performance PeLEDs; there are still some unclear mechanisms that may be in effect at the PEDOT:PSS/LHP NCs interface.

Compared to chloride-based NCs and bromide-based QDs, the LHP NPLs with accurate management of the emissive wavelength are regarded as a candidate for blue PeLEDS. ${ }^{35,39,82}$ In colloidal solution, the prepared LHP NPLs prefer to stack together because of the much larger lateral size compared to the thickness, which leads to a low luminous efficiency due to the re-absorption and rough film morphology caused by the large size of accumulated particles (Fig. 4c). ${ }^{39,82}$ Better dispersion of LHP NPLs in colloidal solution with high concentration and low passivating ligand ratio is key to realizing highperformance PeLEDs. Alternatively, a continuous and uniform LHP NPL film can be prepared using in situ deposition of the precursor solution. ${ }^{35}$ The emissive film formed by tiled LHP NPLs induced a much higher driving voltage in PeLEDs compared to that for LHP NC emitters (Fig. 4b). ${ }^{35}$ Owing to the charge carrier transport in the plane of the $\left[\mathrm{PbX}_{6}\right]^{4-}$ layer being easier compared to the interlayer charge carrier transfer, an emissive layer composed of a closed-packed arrangement of LHP NPLs in a standing position could be used to eliminate the high energy barrier for charge carrier injection, while keeping the light-emission features of the LHP NPLs.

\section{Stability of LHP nanocrystals and PeLEDs}

Although the maximum luminous efficiency achieved for LEDs using LHP NCs is comparable to OLEDs and QLEDs, there are quite a few tough challenges for stability study, including emit- ters and devices. ${ }^{1-24}$ Slow progress may give rise to questions about the prospects of PeLEDs in commercial applications. The degradation of LHPs and PeLEDs are linked to several intrinsic and extrinsic factors, including ion migration, temperature, oxygen, moisture, light and so on. ${ }^{57,110,111}$ In addition, the synergy of multiple factors, e.g., oxygen-caused photodegradation, may multiply the negative influence. ${ }^{110,111}$ Because of the highly ionic bonding, LHPs show a diminished stability against solvent with high dielectric constant, resulting in an ultra-sensitivity to the ambient moisture; thus, a high quality encapsulation is essential for PeLEDs and other LHP applications. ${ }^{57,100}$ Normally, PeLEDs performance measured inside a $\mathrm{N}_{2}$ atmosphere glove box is higher compared to devices, with crude encapsulation, measured in the ambient atmosphere. With consideration of a similar structure, fabrication, and working condition for PeLEDs in comparison to OLEDs and QLEDs, the short-lived lifetime of PeLEDs is supposed to be related to the degradation of LHP NCs and their potential for triggering damage to adjacent functional layers.

With great defect tolerance, the LHP NCs demonstrate superior performance in terms of optical and electronic properties, which does not mean that there is no defect and resulting negative influences. ${ }^{57-59,77}$ As mentioned above, the ion migration, observed in many perovskite-type compounds with the same formula $\mathrm{ABX}_{3}$ but with different components, is related to the point defect in LHPs and is an important consideration for operation stability in applications. ${ }^{112}$ The $J-V$ hysteresis and low stability of solar cells with polymorph LHPs is believed to be caused by the migration of ions and resulting defects at grain boundary. ${ }^{57,107}$ Though capping ligands can suppress the ion migration in LHP NCs, the aggregation of LHP NCs with unstable and dynamic ligand binding suggests that the blocking of ligands is not sufficient, especially in deposited solid films. Thus, matrices have been adopted to surround the LHP NCs for realizing enhanced suppression of ion migration. ${ }^{22,23,92,107,108}$ In LHP NPLs, it was proved that the strong confinement of the $2 \mathrm{D}$ structure provides an effective suppression of ion migration, leading to an improved operation stability for PeLEDs. ${ }^{112}$ Additionally, in hybrid LHPs, organic cations with strong $\mathrm{H}$-bonding and highly constrained motion, e.g., formamidinium, lead to suppressed migration of halogens. ${ }^{113}$

In most high performance PeLEDs, the predominating structure for LHP emitters is the cubic phase; ${ }^{20,21,24}$ however, the transition from the cubic phase to orthorhombic phase is facile, which is supposed to be one reason for the degradation of LHP photovoltaic devices. ${ }^{114,115}$ Though no solid evidence exists yet, the facile phase transition of LHP NCs facilitated by Joule heating may play a non-negligible role in determining the fast decay of resulting PeLEDs. In addition, the LHP NCs and other functional layers, especially the organic semiconductors, are also sensitive to the ambient atmosphere. In contrast, using a matrix with a matched energy level and bipolar charge carrier transport to surround the LHP NCs as the emissive layer can be considered as another approach for improving device stability. 


\section{Conclusion and prospects}

Exciting progress has been achieved for PeLEDs in a rapid timeframe over the past few years, especially using LHP NCs as emitters with the characteristics of defect-tolerant high luminous efficiency with tunable narrow-band emission. The visual experience and luminous efficiency of PeLEDs is no longer a barrier for display applications. However, the low device operation stability and lead toxicity has added uncertainty to the prospects for PeLEDs. Though lead-free LHP alternatives exhibit high PLQYs, progress for PeLEDs fabricated using such materials is still falling far behind lead-based devices. Most importantly, ways to improve the stability of LHP NCs, especially under an applied electrical field, without any negative impact on other superior features is urgently needed for subsequent work.

\section{Conflicts of interest}

There are no conflicts to declare.

\section{Acknowledgements}

This research is supported by the National Research Foundation, Prime Minister's Office, Singapore under its competitive Research Programme (CRP Award No. NRF-CRP142014-03). H. V. D. gratefully acknowledges the financial support from the NRF Investigatorship grant NRF-NRFI201608 and additional support from TUBA.

\section{References}

1 Z. K. Tan, R. S. Moghaddam, M. L. Lai, P. Docampo, R. Higler, F. Deschler, M. Price, A. Sadhanala, L. M. Pazos, D. Credgington, F. Hanusch, T. Bein, H. J. Snaith and R. H. Friend, Nat. Nanotechnol., 2014, 9, 687-692.

2 J. Song, T. Fang, J. Li, L. Xu, F. Zhang, B. Han, Q. Shan and H. Zeng, Adv. Mater., 2018, 1805409.

3 H. Cho, S. Jeong, M. Park, Y. Kim, C. Wolf, C. Lee, J. H. Heo, A. Sadhanala, N. Myoung, S. Yoo, S. H. Im, R. H. Friend and T. W. Lee, Science, 2015, 350, 1222-1225.

4 Y. Sun, L. Zhang, N. Wang, S. Zhang, Y. Cao, Y. Miao, M. Xu, H. Zhang, H. Li, C. Yi, J. Wang and W. Huang, npj Flexible Electron., 2018, 2, 12.

5 N. Wang, L. Cheng, R. Ge, S. Zhang, Y. Miao, W. Zou, C. Yi, Y. Sun, Y. Cao, R. Yang, Y. Wei, Q. Guo, Y. Ke, M. Yu, Y. Jin, Y. Liu, Q. Ding, D. Di, L. Yang, G. Xing, H. Tian, C. Jin, F. Gao, R. H. Friend, J. Wang and W. Huang, Nat. Photonics, 2016, 10, 699-704.

6 J. Mao, H. Lin, F. Ye, M. Qin, J. M. Burkhartsmeyer, H. Zhang, X. Lu, K. S. Wong and W. C. H. Choy, ACS Nano, 2018, 12, 10486-10492.

7 X. Zhang, H. Lin, H. Huang, C. Reckmeier, Y. Zhang, W. C. H. Choy and A. L. Rogac, Nano Lett., 2016, 16, 1415.
8 H. Wang, H. Yu, W. Xu, Z. Yuan, Z. Yan, C. Wang, X. Liu, M. Fahlman, J. Liu, X. Liu and F. Gao, J. Mater. Chem. C, 2018, 6, 6996-7002.

9 Y. Zou, Q. Huang, Y. Yang, M. Ban, S. Li, Y. Han, T. Wu, Y. Tan, X. Gao, T. Song and B. Sun, Adv. Mater. Interfaces, 2018, 5, 1801030.

10 X. Liu, X. Guo, Y. Lv, Y. Hu, Y. Fan, J. Lin, X. Liu and X. Liu, Adv. Opt. Mater., 2018, 1801245.

11 E. Yao, Z. Yang, L. Meng, P. Sun, S. Dong, Y. Yang and Y. Yang, Adv. Mater., 2017, 1606859.

12 Z. Wang, F. Wang, W. Sun, R. Ni, S. Hu, J. Liu, B. Zhang, A. Alsaed, T. Hayat and Z. Tan, Adv. Funct. Mater., 2018, 1804187.

13 B. Zhu, H. Li, J. Ge, H. Li, Y. Yin, K. Wang, C. Chen, J. Yao, Q. Zhang and H. Yao, Nanoscale, 2018, 10, 1926219271.

14 S. Yuan, Z. K. Wang, M. P. Zhuo, Q. S. Tian, Y. Jin and L. S. Liao, ACS Nano, 2018, 12, 9541-9548.

15 H. Huang, H. Lin, S. V. Kershaw, A. S. Susha, W. C. H. Choy and A. L. Rogach, J. Phys. Chem. Lett., 2016, 7, 4398.

16 J. Xing, F. Yan, Y. Zhao, S. Chen, H. Yu, Q. Zhang, R. Zeng, H. V. Demir, X. Sun, A. Huan and Qi. Xiong, ACS Nano, 2016, 10, 6623-6630.

17 J. Wang, C. Song, Z. He, C. Mai, G. Xie, L. Mu, Y. Cun, J. Li, J. Wang, J. Peng and Y. Cao, Adv. Mater., 2018, 1804137.

18 Z. Xiao, R. A. Kerner, L. Zhao, N. L. Tran, K. M. Lee, T. Koh, G. D. Scholes and B. P. Rand, Nat. Photonics, 2017, 11, 108-115.

19 L. Zhang, X. Yang, Q. Jiang, P. Wang, Z. Yin, X. Zhang, H. Tan, Y. Yang, M. Wei, B. R. Sutherland, E. H. Sargent and J. You, Nat. Commun., 2017, 8, 15640.

20 F. Yan, J. Xing, G. Xing, L. Quan, S. T. Tan, J. Zhao, R. Su, L. Zhang, S. Chen, Y. Zhao, A. Huan, E. H. Sargent, Q. Xiong and H. V. Demir, Nano Lett., 2018, 18, 31573164.

21 T. Chiba, Y. Hayashi, H. Ebe, K. Hoshi, J. Sato, S. Sato, Y. Pu, S. Ohisa and J. Kido, Nat. Photonics, 2018, 12, 681687.

22 K. Lin, J. Xing, L. N. Quan, F. P. G. Arquer, X. Gong, J. Lu, L. Xie, W. Zhao, D. Zhang, C. Yan, W. Li, X. Liu, Y. Lu, J. Kirman, E. H. Sargent, Q. Xiong and Z. Wei, Nature, 2018, 562, 245-248.

23 B. Zhao, S. Bai, V. Kim, R. Lamboll, R. Shivanna, F. Auras, J. M. Richter, L. Yang, L. Dai, M. Alsari, X. She, L. Liang, J. Zhang, S. Lilliu, P. Gao, H. J. Snaith, J. Wang, N. C. Greenham, R. H. Friend and D. Di, Nat. Photonics, 2018, 12, 783-789.

24 Y. Cao, N. Wang, H. Tian, J. Guo, Y. Wei, H. Chen, Y. Miao, W. Zou, K. Pan, Y. He, H. Cao, Y. Ke, M. Xu, Y. Wang, M. Yang, K. Du, Z. Fu, D. Kong, D. Dai, Y. Jin, G. Li, H. Li, Q. Peng, J. Wang and W. Huang, Nature, 2018, 562, 249-253.

25 S. Wang, C. Bi, J. Yuan, L. Zhang and J. Tian, ACS Energy Lett., 2018, 3, 245-251. 
26 L. Protesescu, S. Yakunin, M. I. Bodnarchuk, F. Krieg, R. Caputo, C. H. Hendon, R. X. Yang, A. Walsh and M. V. Kovalenko, Nano Lett., 2015, 15, 3692-3696.

27 F. Liu, Y. Zhang, C. Ding, S. Kobayashi, T. Izuishi, N. Nakazawa, T. Toyoda, T. Ohta, S. Hayase, T. Minemoto, K. Yoshino, S. Dai and Q. Shen, ACS Nano, 2017, 11, 10373-10383.

28 B. A. Koscher, Jo. K. Swabeck, N. D. Bronstein and A. P. Alivisatos, J. Am. Chem. Soc., 2017, 139, 6566-6569.

29 J. Butkus, P. Vashishtha, K. Chen, J. K. Gallaher, S. K. K. Prasad, D. Z. Metin, G. Laufersky, N. Gaston, J. E. Halpert and J. M. Hodgkiss, Chem. Mater., 2017, 29, 3644-3652.

30 F. Zhang, H. Zhong, C. Chen, X. Wu, X. Hu, H. Huang, J. Han, B. Zou and Y. Dong, ACS Nano, 2015, 9, 45334542.

31 Y. H. Kima, H. Choa and T. W. Lee, Proc. Natl. Acad. Sci. U. S. A., 2016, 113, 11694-11702.

32 G. Lozano, J. Phys. Chem. Lett., 2018, 9, 3987-3997.

33 M. V. Kovalenko, L. Protesescu and M. I. Bodnarchuk, Science, 2017, 358, 745-750.

34 X. Du, G. Wu, J. Cheng, H. Dang, K. Ma, Y. Zhang, P. Tan and S. Chen, RSC Adv., 2017, 7, 10391-10396.

35 J. Xing, Y. Zhao, M. Askerka, L. N. Quan, X. Gong, W. Zhao, J. Zhao, H. Tan, G. Long, L. Gao, Z. Yang, O. Voznyy, J. Tang, Z. H. Lu, Q. Xiong and E. H. Sargent, Nat. Commun., 2018, 9, 3541.

36 A. Perumal, S. Shendre, Mi. Li, Y. K. E. Tay, V. K. Sharma, S. Chen, Z. Wei, Q. Liu, Y. Gao, P. J. S. Buenconsejo, S. T. Tan, C. L. Gan, Q. Xiong, T. C. Sum and H. V. Demir, Sci. Rep., 2016, 6, 36733.

37 M. R. Leyden, L. Meng, Y. Jiang, L. K. Ono, L. Qiu, E. J. Juarez-Perez, C. Qin, C. Adachi and Y. Qi, J. Phys. Chem. Lett., 2017, 8, 3193-3198.

38 H. C. Wang, Z. Bao, H. Y. Tsai, A. C. Tang and R. S. Liu, Small, 2018, 14, 1702433.

39 J. A. Sichert, Y. Tong, N. Mutz, M. Vollmer, S. Fischer, K. Z. Milowska, R. G. Cortadella, B. Nickel, C. CardenasDaw, J. K. Stolarczyk, A. S. Urban and J. Feldmann, Nano Lett., 2015, 15, 6521-6527.

40 G. Xing, N. Mathews, S. S. Lim, N. Yantara, X. Liu, D. Sabba, M. Grätzel, S. Mhaisalkar and T. C. Sum, Nat. Mater., 2014, 13, 476-480.

41 M. B. Johnston and L. M. Herz, Acc. Chem. Res., 2016, 49, 146-154.

42 G. Xing, B. Wu, X. Wu, M. Li, B. Du, Q. Wei, J. Guo, E. K. L. Yeow, T. C. Sum and W. Huang, Nat. Commun., 2017, 8, 14558.

43 F. Ambrosio, J. Wiktor, F. D. Angelisbc and A. Pasquarelloa, Energy Environ. Sci., 2018, 11, 101-105.

44 P. Azarhoosh, S. McKechnie, J. M. Frost, A. Walsh and M. Schilfgaarde, APL Mater., 2016, 4, 091501.

45 V. D’Innocenzo, G. Grancini, M. J. P. Alcocer, A. R. S. Kandada, S. D. Stranks, M. M. Lee, G. Lanzani, H. J. Snaith and A. Petrozza, Nat. Commun., 2016, 5, 3586 .
46 S. D. Stranks, R. L. Z. Hoye, D. Di, R. H. Friend and F. Deschler, Adv. Mater., 2018, 1803336.

47 A. Paulke, S. D. Stranks, J. Kniepert, J. Kurpiers, C. M. Wolff, N. Schon, H. J. Snaith, T. J. K. Brenner and D. Neher, Appl. Phys. Lett., 2016, 108, 113505.

48 Q. Wang, X. Liu, Y. Qiu, K. Chen, L. Zhou and Q. Wang, AIP Adv., 2018, 8, 025108.

49 Q. Li and T. Lian, J. Phys. Chem. Lett., 2019, 10, 566573.

50 D. B. Straus and C. R. Kagan, J. Phys. Chem. Lett., 2018, 9, 1434-1447.

51 H.-H. Fang, L. Protesescu, D. M. Balazs, S. Adjokatse, M. V. Kovalenko and M. A. Loi, Small, 2017, 13, 1700673.

52 C. B. Lan, C. Li, X. Zhang, X. Huang and X. M. Wang, Nano Lett., 2018, 18, 2074-2080.

53 M. Lorenzon, L. Sortino, Q. Akkerman, S. Accornero, J. Pedrini, M. Prato, V. Pinchetti, F. Meinardi, L. Manna and S. Brovelli, Nano Lett., 2017, 17, 3844-3853.

54 M. Fu, P. Tamarat, H. Huang, J. Even, A. L. Rogach and B. Lounis, Nano Lett., 2017, 17, 2895-2901.

55 Z. Yang, A. Surrente, K. Galkowski, N. Bruyant, D. K. Maude, A. A. Haghighirad, H. J. Snaith, P. Plochocka and R. J. Nicholas, J. Phys. Chem. Lett., 2017, 8, 18511855.

56 Y. Wang and H. Sun, Small Methods, 2018, 2, 1700252.

57 H. Huang, M. I. Bodnarchuk, S. V. Kershaw, M. V. Kovalenko and A. L. Rogach, ACS Energy Lett., 2017, 2, 2071-2083.

58 J. Kang and L. W. Wang, J. Phys. Chem. Lett., 2017, 8, 489493.

59 H. Tan, F. Che, M. Wei, Y. Zhao, M. I. Saidaminov, P. Todorović, D. Broberg, G. Walters, F. Tan, T. Zhuang, B. Sun, Z. Liang, H. Yuan, E. Fron, J. Kim, Z. Yang, O. Voznyy, M. Asta and E. H. Sargent, Nat. Commun., 2018, 9, 3100 .

60 S. D. Stranks, ACS Energy Lett., 2017, 2, 1515.

61 J. M. Ball and A. Petrozza, Nat. Energy, 2016, 1, 16149.

62 R. J. Stoddard, A. Rajagopal, R. L. Palmer, I. L. Braly, A. K. Y. Jen and H. W. Hillhouse, ACS Energy Lett., 2018, 3, 1261-1268.

63 F. Deschler, M. Price, S. Pathak, L. E. Klintberg, D. D. Jarausch, R. Higler, S. Huttner, T. Leijtens, S. D. Stranks, H. J. Snaith, M. Atature, R. T. Phillips and R. H. Friend, J. Phys. Chem. Lett., 2014, 5, 1421.

64 M. Abdi-Jalebi, Z. Andaji-Garmaroudi, S. Cacovich, C. Stavrakas, B. Philippe, J. M. Richter, M. Alsari, E. P. Booker, E. M. Hutter, A. J. Pearson, S. Lilliu, T. J. Savenije, H. Rensmo, G. Divitini, C. Ducati, R. H. Friend and S. D. Stranks, Nature, 2018, 555, 497.

65 I. L. Braly, D. W. deQuilettes, L. M. Pazos-Outón, S. Burke, M. E. Ziffer, D. S. Ginger and H. W. Hillhouse, Nat. Photonics, 2018, 12, 355.

66 R. Brenes, D. Guo, A. Osherov, N. K. Noel, C. Eames, E. M. Hutter, S. K. Pathak, F. Niroui, R. H. Friend, M. S. Islam, H. J. Snaith, V. Bulović, T. J. Savenije and S. D. Stranks, Joule, 2017, 1, 155. 
67 N. Kawano, M. Koshimizu and K. Asai, J. Phys. Chem. C, 2012, 116, 22992-22995.

68 M. A. Becker, R. Vaxenburg, G. Nedelcu, P. C. Sercel, A. Shabaev, M. J. Mehl, J. G. Michopoulos, S. G. Lambrakos, N. Bernstein, J. L. Lyons, T. Stöferle, R. F. Mahrt, M. V. Kovalenko, D. J. Norris, G. Rainò and A. L. Efros, Nature, 2018, 553, 189-193.

69 X. Li, Y. Wu, S. Zhang, B. Cai, Y. Gu, J. Song and H. Zeng, Adv. Funct. Mater., 2016, 26, 2435-2445.

70 Y. H. Suh, T. Kim, J. W. Choi, C. L. Lee and J. Park, ACS Appl. Nano Mater., 2018, 1, 488-496.

71 S. Govinda, B. P. Kore, D. Swain, A. Hossain, C. De, T. N. Row and D. D. Sarma, J. Phys. Chem. C, 2018, 122, 13758-13766.

72 X. Liang, R. W. Baker, K. Wu, W. Deng, D. Ferdani, P. S. Kubiak, F. Marken, L. Torrente-Murciano and P. J. Cameron, React. Chem. Eng., 2018, 3, 640-644.

73 S. Govinda, B. P. Kore, M. Bokdam, P. Mahale, A. Kumar, S. Pal, B. Bhattacharyya, J. Lahnsteiner, G. Kresse, C. Franchini, A. Pandey and D. D. Sarma, J. Phys. Chem. Lett., 2017, 8, 4113-4121.

74 A. D. Wright, C. Verdi, R. L. Milot, G. E. Eperon, M. A. Pérez-Osorio, H. J. Snaith, F. Giustino, M. B. Johnston I and L. M. Herz, Nat. Commun., 2016, 7, 11755.

75 G.-J. A. H. Wetzelaer, M. Scheepers, A. M. Sempere, C. Momblona, J. Ávila and H. J. Bolink, Adv. Mater., 2015, 27, 1837-1841.

76 K. H. Wang, L. C. Li, M. Shellaiah and K. W. Sun, Sci. Rep., 2017, 7, 13643.

77 R. E. Brandt, J. R. Poindexter, P. Gorai, R. C. Kurchin, R. L. Z. Hoye, L. Nienhaus, M. W. B. Wilson, J. A. Polizzotti, R. Sereika, R. Žaltauskas, L. C. Lee, J. L. MacManus-Driscoll, M. Bawendi, V. Stevanovic and T. Buonassisi, Chem. Mater., 2017, 29, 4667-4674.

78 S. Draguta, O. Sharia, S. J. Yoon, M. C. Brennan, Y. V. Morozov, J. S. Manser, P. V. Kamat, W. F. Schneider and M. Kuno, Nat. Commun., 2017, 8, 200.

79 M. C. Brennan, S. Draguta, P. V. Kamat and M. Kuno, ACS Energy Lett., 2018, 3, 204-213.

80 P. Vashishtha and J. E. Halpert, Chem. Mater., 2017, 29, 5965-5973.

81 A. A. Lohar, A. Shinde, R. Gahlaut, A. Sagdeo and S. Mahamuni, J. Phys. Chem. C, 2018, 122, 25014-25020.

82 B. J. Bohn, Y. Tong, M. Gramlich, M. L. Lai, M. Gramlich, K. Wang, R. L. Z. Hoye, P. M. Buschbaum, S. D. Stranks, A. S. Urban, L. Polavarapu and J. Feldmann, Nano Lett., 2018, 18, 5231-5238.

83 M. C. Weidman, M. Seitz, S. D. Stranks and W. A. Tisdale, ACS Nano, 2016, 10, 7830-7839.

84 Q. A. Akkerman, S. G. Motti, A. Kandada, E. Mosconi, V. D’Innocenzo, G. Bertoni, S. Marras, B. A. Kamino, L. Miranda, F. D. Angelis, A. Petrozza, M. Prato and L. Manna, J. Am. Chem. Soc., 2016, 138, 1010-1016.

85 K. Miyata, D. Meggiolaro, M. T. Trinh, P. P. Joshi, E. Mosconi, S. C. Jones, F. De Angelis and X. Y. Zhu, Sci. Adv., 2017, 3, 1701217.
86 P. Calado, A. M. Telford, D. Bryant, X. Li, J. Nelson, B. C. O'Regan and P. R. F. Barnesb, Nat. Commun., 2016, 7, 13831.

87 W. Tress, J. Phys. Chem. Lett., 2017, 8, 3106-3114.

88 J. Huang, Y. Wu, Z. Zhu, W. Y. Shih and W. Shih, Chem. Phys. Lett., 2018, 702, 21-25.

89 Y. Wu, C. Wei, X. Li, Y. Li, S. Qiu, W. Shen, B. Cai, Z. Sun, D. Yang, Z. Deng and H. Zeng, ACS Energy Lett., 2018, 3, 2030-2037.

90 Y. Dong, T. Qiao, D. Kim, D. Parobek, D. Rossi and D. H. Son, Nano Lett., 2018, 18, 3716-3722.

91 Y. Bekenstein, B. A. Koscher, S. W. Eaton, P. Yang and A. P. Alivisatos, J. Am. Chem. Soc., 2015, 137, 16008-16011.

92 G. Li, Z. Tan, D. Di, M. L. Lai, L. Jiang, J. Lim, R. H. Friend and N. C. Greenham, Nano Lett., 2015, 15, 2640-2644.

93 J. Sun, J. Yang, J. I. Lee, J. H. Cho and M. S. Kang, J. Phys. Chem. Lett., 2018, 9, 1573-1583.

94 A. H. Slavney, T. Hu, A. M. Lindenberg and H. I. Karunadasa, J. Am. Chem. Soc., 2016, 138, 2138.

95 M. Leng, Y. Yang, K. Zeng, Z. Chen, Z. Tan, S. Li, J. Li, B. Xu, D. Li, P. H. Matthew, Y. Fu, T. Zhai, L. Xu, G. Niu, S. Jin and J. Tang, Adv. Funct. Mater., 2017, 28, 1704446.

96 J. Zhang, Y. Yang, H. Deng, U. Farooq, X. Yang, J. Khan, J. Tang and H. Song, ACS Nano, 2017, 11, 9294.

97 J. Luo, X. Wang, S. Li, J. Liu, Y. Guo, G. Niu, L. Yao, Y. Fu, L. Gao, Q. Dong, C. Zhao, M. Leng, F. Ma, W. Liang, L. Wang, S. Jin, J. Han, L. Zhang, J. Etheridge, J. Wang, Y. Yan, E. H. Sargent and J. Tang, Nature, 2018, 563, 541545.

98 M. Leng, Z. Chen, Y. Yang, Z. Li, K. Zeng, K. Li, G. Niu, Y. He, Q. Zhou and J. Tang, Angew. Chem., Int. Ed., 2016, 55, 15012.

99 J. Zhang, Y. Yang, H. Deng, U. Farooq, X. Yang, J. Khan, J. Tang and H. Song, ACS Nano, 2017, 11, 9294.

100 J. D. Roo, M. Ibánez, P. Geiregat, G. Nedelcu, W. Walravens, J. Maes, J. C. Martins, I. V. Driessche, M. V. Kovalenko and Z. Hens, ACS Nano, 2016, 10, 20712081.

101 X. Shan, J. Li, M. Chen, T. Geske, S. G. R. Bade and Z. Yu, J. Phys. Chem. Lett., 2017, 8, 2412-2419.

102 S. Zhang, M. Stolterfoht, A. Armin, Q. Lin, F. Zu, J. Sobus, H. Jin, N. Koch, P. Meredith, P. L. Burn and D. Neher, ACS Appl. Mater. Interfaces, 2018, 10, 21681-21687.

103 J. S. Park, J. Kyhm, H. H. Kim, S. Jeong, J. Kang, S. Lee, K. T. Lee, K. Park, N. Barange, J. Han, J. D. Song, W. K. Choi and K. Han II, Nano Lett., 2016, 16, 69466953.

104 V. R. Nikitenko, A. Yu. Saunina, A. P. Tyutnev and O. V. Prezhdo, J. Phys. Chem. C, 2017, 121, 7776-7781.

105 H. Mu, I. Reddy, J. Hunt, P. Severs and S. Patil, J. Phys. D: Appl. Phys., 2010, 43, 195103.

106 B. R. Sutherland and E. H. Sargent, Nat. Photonics, 2016, 10, 295-302.

107 Y. H. Kim, C. Wolf, H. Kim and T. W. Lee, Nano Energy, 2018, 52, 329-335. 
108 Y. Xin, H. Zhao and J. Zhang, ACS Appl. Mater. Interfaces, 2018, 10, 4971-4980.

109 T. Cheng, Y. Zhang, J. Zhang, W. Lai and W. Huang, J. Mater. Chem. A, 2016, 4, 10493-10499.

110 E. J. Juarez-Perez, Z. Hawash, S. R. Raga, L. K. Ono and Y. Qi, Energy Environ. Sci., 2016, 9, 3406-3410.

111 B. Conings, J. Drijkoningen, N. Gauquelin, A. B. Jan D'Haen, L. D'Olieslaeger, A. Ethirajan, J. Verbeeck, J. Manca, E. Mosconi, F. D. Angelis and H. G. Boyen, Adv. Energy Mater., 2015, 5, 1500477.
112 U. Giovanella, M. Pasini, M. Lorenzon, F. Galeotti, C. Lucchi, F. Meinardi, S. Luzzati, B. Dubertret and S. Brovelli, Nano Lett., 2018, 18, 3441-3448.

113 A. Oranskaia, J. Yin, O. M. Bakr, J. L. Brédas and O. F. Mohammed, J. Phys. Chem. Lett., 2018, 9, 5474-5480.

114 R. J. Sutton, M. R. Filip, A. A. Haghighirad, N. Sakai, B. Wenger, F. Giustino and H. J. Snaith, ACS Energy Lett., 2018, 3, 1787-1794.

115 W. Zhou, F. Sui, G. Zhong, G. Cheng, M. Pan, C. Yang and S. Ruan, J. Phys. Chem. Lett., 2018, 9, 4915-4920. 